\title{
Some remarks on Diophantine approximation by the Jacobi-Perron algorithm
}

by

\section{FRITZ SchweIGER (Salzburg)}

Dedicated to Wolfgang Schmidt on the occasion of his 75th birthday

1. Introduction. In an attempt to generalize Lagrange's theorem to cubic numbers Jacobi [2] in a classical paper proposed a new algorithm. Perron [5] extended this algorithm to arbitrary dimension $d$. An easy way to describe the algorithm is as follows:

Let $d \geq 2$ and $E^{d}=\left\{x=\left(x_{1}, \ldots, x_{d}\right): 0 \leq x_{i}<1, i=1, \ldots, d\right\}$. If $x_{1}=0$ then the map $T$ is not defined (the algorithm stops). If $x_{1} \neq 0$ we define the map $T: E^{d} \rightarrow E^{d}$ as

$$
\begin{gathered}
T x=\left(\frac{x_{2}}{x_{1}}-a_{1}, \frac{x_{3}}{x_{1}}-a_{2}, \ldots, \frac{1}{x_{1}}-a_{d}\right), \\
a_{1}:=\left[x_{2} / x_{1}\right], \quad a_{2}:=\left[x_{3} / x_{1}\right], \ldots, a_{d}:=\left[1 / x_{1}\right],
\end{gathered}
$$

and then we iterate. We define

$$
a=a(x)=\left(a_{1}, \ldots, a_{d}\right) .
$$

The sequence of integral vectors $a^{(n)}=a\left(T^{n-1} x\right)$ satisfies the so-called Perron conditions (Schweiger [8]):

(i) $0 \leq a_{s}^{(t)} \leq a_{d}^{(t)}, 1 \leq s \leq d-1 ; a_{d}^{(t)} \geq 1$.

(ii) The relations $(0 \leq R \leq s-1)$

$$
a_{s}^{(t)}=a_{d}^{(t)}, \ldots, a_{s-R}^{(t+R)}=a_{d-R}^{(t+R)}
$$

imply

$$
a_{s-R-1}^{(t+R+1)} \leq a_{d-R-1}^{(t+R+1)} .
$$

For $s=R+1$ we define formally $a_{0}^{(t+R+1)}=0$.

Key words and phrases: multidimensional continued fractions, periodic expansions. 
If the algorithm does not stop we write the expansion of the point $x$ in the form

$$
x=\left(\begin{array}{cccc}
a_{1}^{(1)} & a_{1}^{(2)} & a_{1}^{(3)} & \ldots \\
a_{2}^{(1)} & a_{2}^{(2)} & a_{2}^{(3)} & \ldots \\
\cdots \ldots & \ldots \ldots & \ldots \\
a_{d}^{(1)} & a_{d}^{(2)} & a_{d}^{(3)} & \ldots
\end{array}\right)
$$

As usual a periodic algorithm will be indicated by a horizontal bar over the matrix. Further an infinite sequence of matrices

$$
\beta\left(a^{(n)}\right)=\left(\begin{array}{ccccc}
a_{d}^{(n)} & 0 & \ldots & 0 & 1 \\
1 & 0 & \ldots & 0 & 0 \\
a_{1}^{(n)} & 1 & \ldots & 0 & 0 \\
\ldots \ldots & \ldots & \ldots & \ldots & \ldots \\
a_{d-1}^{(n)} & 0 & \ldots & 1 & 0
\end{array}\right), \quad n=1,2, \ldots
$$

is produced.

We introduce the matrices $\beta\left(a^{(1)}, \ldots, a^{(n)}\right)=\beta\left(a^{(1)}\right) \circ \cdots \circ \beta\left(a^{(n)}\right)$ and we define numbers $A_{i}^{(j)}$ by the equation

$$
\beta\left(a^{(1)}, \ldots, a^{(n)}\right)=\left(\begin{array}{cccc}
A_{0}^{(d+n+1)} & A_{0}^{(n+1)} & \ldots & A_{0}^{(n+d)} \\
A_{1}^{(d+n+1)} & A_{1}^{(n+1)} & \ldots & A_{1}^{(n+d)} \\
\ldots \ldots \ldots \ldots & \ldots \ldots \ldots & \ldots & \ldots \ldots \\
A_{d}^{(d+n+1)} & A_{d}^{(n+1)} & \ldots & A_{d}^{(n+d)}
\end{array}\right) .
$$

It is convenient to add the unit matrix as

$$
\mathbf{1}=\left(\begin{array}{cccc}
A_{0}^{(d+1)} & A_{0}^{(1)} & \ldots & A_{0}^{(d)} \\
A_{1}^{(d+1)} & A_{1}^{(1)} & \ldots & A_{1}^{(d)} \\
\ldots \ldots \ldots & \ldots \ldots \ldots & \ldots \\
A_{d}^{(d+1)} & A_{d}^{(1)} & \ldots & A_{d}^{(d)}
\end{array}\right)
$$

Then we have the recursion relations

$$
A_{i}^{(n+d+1)}=A_{i}^{(n)}+a_{1}^{(n)} A_{i}^{(n+1)}+\cdots+a_{d}^{(n)} A_{i}^{(d+n)}, \quad i=0,1, \ldots, d .
$$

It is easy to see (Schweiger [8]) that if $y=T^{n} x$ then

$$
x_{i}=\frac{A_{i}^{(n+d+1)}+A_{i}^{(n+1)} y_{1}+\cdots+A_{i}^{(n+d)} y_{d}}{A_{0}^{(n+d+1)}+A_{0}^{(n+1)} y_{1}+\cdots+A_{0}^{(n+d)} y_{d}}, \quad 1 \leq i \leq d .
$$

One of the ideas of considering a multidimensional continued fraction is the expectation that the sequence of rational points

$$
\left(\frac{A_{1}^{(n+d+1)}}{A_{0}^{(n+d+1)}}, \frac{A_{2}^{(n+d+1)}}{A_{0}^{(n+d+1)}}, \ldots, \frac{A_{d}^{(n+d+1)}}{A_{0}^{(n+d+1)}}\right)
$$


should provide "good" Diophantine approximations for $x=\left(x_{1}, \ldots, x_{d}\right)$. In fact, only for $d=2$ is the approximation result

$$
\left|x_{i}-\frac{A_{i}^{(n+3)}}{A_{0}^{(n+3)}}\right|<\frac{1}{A_{0}^{(n+3)}}, \quad i=1,2,
$$

true generally (see Schweiger [8]). Perron [5] and Paley \& Ursell [3] have shown that this result is best possible in the following sense. For every $\delta>0$ there are points $x \in E^{2}$ such that

$$
\max _{i=1,2}\left|x_{i} A_{0}^{(n+3)}-A_{i}^{(n+3)}\right|>\left(A_{0}^{(n+3)}\right)^{-\delta} .
$$

For dimension $d \geq 4$ it has long been known that even

$$
\limsup _{n}\left|x_{i} A_{0}^{(n+3)}-A_{i}^{(n+3)}\right|=\infty
$$

is possible. Paley \& Ursell say that this is also true for $d=3$ but they do not give an example. They state that for $d \geq 3$ there is a constant $\omega=\omega(d)>1$ such that $\left|A_{00}^{(N)} x_{i}-A_{i 0}^{(N)}\right| \ll \omega^{N}$.

The present paper discusses Diophantine properties of simultaneous approximation. Another measure of approximation has been discussed in Schmidt [6] and Schweiger [7]. In Section 2 we first give a new short proof of convergence in dimension $d=2$ and discuss the relation of the eigenvalues of the periodicity matrix to Diophantine properties. In Section 3 we concentrate on $d=3$. It is shown that there exist periodic expansions of period length $p$ such that the second eigenvalue $\varrho_{1}$ is $(-1)^{p}$. A family of examples is given with period length $p=6$ such that all three cases $\varrho_{1}<-1, \varrho_{1}=-1$, $\varrho_{1}>1$ appear. In Section 4 we give an upper bound for the Paley-Ursell constant $\omega=\omega(3)$.

\section{The rate of convergence and eigenvalues}

TheOREM 1. The Jacobi-Perron algorithm is convergent for $d=2$.

Proof. The proof is a combination of two identities. Let

$$
D_{N}=A_{00}^{(N)} x_{i}-A_{i 0}^{(N)}, \quad i=1,2 .
$$

Then the usual recursion relation gives

$$
D_{N+3}=a_{2}^{(N)} D_{N+2}+a_{1}^{(N)} D_{N+1}+D_{N} .
$$

If $y=T^{N} x$ then we have the equation (a special case of Perron's relations; see Schweiger [8])

$$
D_{N+3}=-y_{1} D_{N+1}-y_{2} D_{N+2} .
$$

Let us assume that $D_{N+3}>0$. If $D_{N+2}<0$ and $D_{N+1}<0$ then the recursion relation implies $D_{N+3}<D_{N}$. If $D_{N+2} D_{N+1}<0$ Perron's relation shows 
$D_{N+3} \leq \max \left(y_{1}\left|D_{N+1}\right|, y_{2}\left|D_{N+2}\right|\right) \leq \max \left(\left|D_{N+1}\right|,\left|D_{N+2}\right|\right)$. Therefore in all cases $D_{N+3} \leq \max \left(\left|D_{N+1}\right|,\left|D_{N+2}\right|,\left|D_{N}\right|\right)$.

Next we consider periodic algorithms. If the algorithm is eventually periodic, i.e. $T^{p+m} x=T^{m} x$ for some $p \geq 1, m \geq 0$, then $x_{1}, \ldots, x_{d}$ belong to a number field of degree $\leq d+1$. It is sufficient to consider the case of purely periodic expansions, i.e. $m=0$. Then the periodicity matrix

$$
B:=\beta\left(a^{(1)}, \ldots, a^{(p)}\right)
$$

is attached to the algorithm. Its characteristic polynomial

$$
f(\varrho)=\operatorname{det}(\varrho \mathbf{1}-B)
$$

has the following property (see Schweiger [8]). There is a real simple root $\varrho_{0}>1$ such that $\varrho_{0}>\left|\varrho_{1}\right| \geq\left|\varrho_{2}\right| \geq \cdots \geq\left|\varrho_{d}\right|$ for all other roots $\varrho_{1}, \ldots, \varrho_{d}$. Perron [5] proved that there is an index $i, 1 \leq i \leq d$, such that

$$
\limsup _{n \rightarrow \infty}\left|x_{i} A_{0}^{(p n+d+1)}-A_{i}^{(p n+d+1)}\right| \gg\left|\varrho_{1}\right|^{n} .
$$

Therefore if we find a periodic algorithm with $\left|\varrho_{1}\right|>1$ then the approximation

$$
\left|x_{i}-\frac{A_{i}^{(n+d+1)}}{A_{0}^{(n+d+1)}}\right| \ll \frac{1}{A_{0}^{(n+d+1)}}, \quad i=1, \ldots, d,
$$

cannot be true. Now this is easy and well known for $d \geq 4$. For period length $p=1$ we obtain

$$
f(\varrho)=\varrho^{d+1}-a_{d} \varrho^{d}-\cdots-a_{1} \varrho-1 .
$$

Therefore if we choose $a_{1}, \ldots, a_{d}$ such that $f(-1)<0$ for $d \equiv 1 \bmod 2$ and $f(-1)>0$ for $d \equiv 0 \bmod 2$ then $f(\varrho)$ has a root $\varrho_{1}<-1$. Clearly, one has to control Perron's conditions. For $d=4$ this leads to

$$
a_{3}+a_{1}>2+a_{4}+a_{2},
$$

which is satisfied for $a_{4}=5, a_{3}=a_{1}=4, a_{2}=0$, say. For $d=5$ this leads to

$$
a_{5}+a_{3}+a_{1}<a_{4}+a_{2},
$$

which is satisfied for $a_{5}=3, a_{3}=a_{1}=0, a_{4}=a_{2}=2$, say.

For $d=2$ one can show that the polynomial $f(\varrho)$ is irreducible and furthermore that $\left|\varrho_{2}\right| \leq\left|\varrho_{1}\right|<1$ (see Schweiger [8] and the references given there). However, the root $\varrho_{1}$ can be arbitrarily close to -1 .

Proposition. For every $\varepsilon>0$ there is a periodic algorithm such that $-1<\varrho_{1}<-1+\varepsilon$.

Proof. We consider the periodic algorithm (with period length $p=2$ )

$$
x=\left(\begin{array}{ll}
0 & 1 \\
1 & b
\end{array}\right)
$$


where $b \geq 4$ is sufficiently large. Then

$$
B=\left(\begin{array}{ccc}
b+1 & 1 & 1 \\
b & 0 & 1 \\
1 & 0 & 0
\end{array}\right)
$$

and

$$
f(\varrho)=\varrho^{3}-\varrho^{2}(1+b)-\varrho(1+b)-1 .
$$

Then for fixed $N \geq 2$ we calculate

$$
f\left(-1+\frac{1}{N}\right)>-2+\frac{4+b}{N}-\frac{4+b}{N^{2}} .
$$

If $b=2 N$ then

$$
f\left(-1+\frac{1}{N}\right)=\frac{2 N-4}{N^{2}} \geq 0 .
$$

Therefore $-1<\varrho_{1}<-1+1 / N$.

Remarks. (1) Paley \& Ursell [3] have shown that for periodic Jacobi algorithms in dimension $d=2$ one obtains

$$
\left|x_{i}-\frac{A_{i}^{(p g)}}{A_{0}^{(p g)}}\right| \ll \frac{1}{\left(A_{0}^{(p g)}\right)^{1+\delta}}, \quad i=1,2,
$$

for some $\delta=\delta(x)>0$ and for all integers $g \geq 1$ (see also Schweiger [8]). Here $p$ denotes the length of the period. Since $A_{0}^{(2 n)} \sim \varrho_{0}^{n}$ this result shows that the exponent of approximation cannot be uniformly of order $1+\delta$ with $\delta>0$.

(2) Another important connection between eigenvalues and periodicity has been established by Paysant-Le Roux \& Dubois [4].

3. Remarks on the case $d=3$. Now the interesting question remains what happens in dimension $d=3$. If we choose $a_{3}=a_{2} \geq 1$ and $a_{1}=0$ then the characteristic equation

$$
f(\varrho)=\varrho^{4}-a_{3} \varrho^{3}-a_{2} \varrho^{2}-1
$$

has the root $\varrho_{1}=-1$. Therefore nothing better than

$$
\left|x_{i}-\frac{A_{i}^{(n+4)}}{A_{0}^{(n+4)}}\right| \ll \frac{1}{A_{0}^{(n+4)}}, \quad i=1,2,3,
$$

can be expected. This result can be generalized as follows. 
THEOREM 2. Let

$$
x=\left(\begin{array}{cccc}
0 & 0 & \ldots & 0 \\
a_{2}^{(1)} & a_{2}^{(2)} & \ldots & a_{2}^{(p)} \\
a_{3}^{(1)} & a_{3}^{(2)} & \ldots & a_{3}^{(p)}
\end{array}\right) \quad \text { with } a_{2}^{(i)}=a_{3}^{(i)}, 1 \leq i \leq p
$$

be a periodic Jacobi-Perron algorithm. Then the second characteristic root is

$$
\varrho_{1}=(-1)^{p} .
$$

Proof. It is easy to see that the row $(1,-1,1,-1)$ is an eigenvector of the periodicity matrix $B$. For $p=1$ we put

$$
a_{2}^{(i)}=a_{3}^{(i)}=c
$$

and remark that

$$
(1,-1,1,-1)\left(\begin{array}{cccc}
c & 0 & 0 & 1 \\
1 & 0 & 0 & 0 \\
0 & 1 & 0 & 0 \\
c & 0 & 1 & 0
\end{array}\right)=-(1,-1,1,-1)
$$

Then the result follows by induction.

REMARKs. (1) This theorem can easily be generalized to higher dimensions. If we choose nonnegative integers which satisfy the condition

$$
1-b_{1}+b_{2}-\cdots+(-1)^{n} b_{n}=(-1)^{n+1}
$$

and the so-called Perron conditions, then $\varrho_{1}=-1$ is a root of $f(z)=$ $z^{n+1}-b_{n} z^{n}-\cdots-b_{1} z-1=0$. Then in a similar way one can construct periodic expansions of length $p$ with eigenvalue $\varrho_{1}=(-1)^{p}$. The row $(1,1,-1,1,-1, \ldots, 1,-1)$ is an eigenvector of the periodicity matrix if $d$ is even, and $(1,-1,1,-1, \ldots, 1,-1)$ is an eigenvector if $d$ is odd.

(2) Note that this shows that the characteristic polynomial $f(\varrho)$ can be reducible for $d \geq 3$.

(3) By simple calculations one finds another type of equations with $\varrho_{1}=$ -1 , namely the points with period length $p=2$

$$
x=\left(\begin{array}{cc}
a_{1}^{(1)} & a_{1}^{(2)} \\
0 & a_{2}^{(2)} \\
a_{3}^{(1)} & a_{3}^{(2)}
\end{array}\right)
$$

with the conditions $a_{1}^{(1)}=a_{3}^{(1)}$, (by Perron's conditions) $a_{2}^{(2)} \geq 1$, and $a_{3}^{(2)}>a_{1}^{(2)}$. It is in fact easy to show that for period length $p \leq 2$ the case 
$\left|\varrho_{1}\right|>1$ does not occur at all and $\left|\varrho_{1}\right|=1$ can only happen in the cases just described (see e.g. Dubois, Farhane \& Paysant-Le Roux [1]).

(4) A mixture of both types does not give an algorithm with similar properties. Take

$$
x=\left(\begin{array}{lll}
1 & 0 & 0 \\
0 & 1 & 1 \\
1 & 2 & 1
\end{array}\right) .
$$

Then the periodicity matrix is

$$
B=\left(\begin{array}{llll}
4 & 1 & 1 & 3 \\
3 & 1 & 1 & 2 \\
4 & 0 & 1 & 3 \\
1 & 0 & 0 & 0
\end{array}\right)
$$

but $f(-1)=4>0$ and $f(1)=-14<0$.

THEOREM 3.

(i) The periodic expansion

$$
x=\left(\begin{array}{cccc}
a_{1}^{(1)} & a_{1}^{(2)} & a_{1}^{(3)} & a_{1}^{(4)} \\
0 & a_{2}^{(2)} & 0 & a_{2}^{(4)} \\
a_{3}^{(1)} & a_{3}^{(2)} & a_{3}^{(3)} & a_{3}^{(4)}
\end{array}\right)
$$

with $a_{1}^{(1)}=a_{3}^{(1)}, a_{1}^{(3)}=a_{3}^{(3)}, a_{2}^{(2)} \geq 1, a_{2}^{(4)} \geq 1, a_{3}^{(2)}>a_{1}^{(2)}, a_{3}^{(4)}>a_{1}^{(4)}$ has the eigenvalue $\varrho_{1}=1$.

(ii) For the periodic expansions of type

$$
x=\left(\begin{array}{cccccc}
a_{1}^{(1)} & a_{1}^{(2)} & a_{1}^{(3)} & a_{1}^{(4)} & a_{1}^{(5)} & a_{1}^{(6)} \\
0 & a_{2}^{(2)} & 0 & a_{2}^{(4)} & 0 & a_{2}^{(6)} \\
a_{3}^{(1)} & a_{3}^{(2)} & a_{3}^{(3)} & a_{3}^{(4)} & a_{3}^{(5)} & a_{3}^{(6)}
\end{array}\right)
$$

with $a_{1}^{(2 k-1)}=a_{3}^{(2 k-1)}, a_{2}^{(2 k)} \geq 1, a_{3}^{(2 k)}>a_{1}^{(2 k)}, 1 \leq k \leq 3$, all three cases $\varrho_{1}<-1, \varrho_{1}=-1, \varrho_{1}>1$ can occur.

Proof. (i) With a slightly changed notation we first consider

$$
x=\left(\begin{array}{cccc}
c_{1} & a_{2} & c_{3} & a_{4} \\
0 & b_{2} & 0 & b_{4} \\
c_{1} & c_{2} & c_{3} & c_{4}
\end{array}\right) .
$$


Then a calculation of the product of the matrices shows that the matrix

$$
B=\left(\begin{array}{cccc}
c_{1} c_{2}+b_{2} & 0 & 1 & c_{1} \\
c_{2} & 0 & 0 & 1 \\
c_{1} c_{2}+1 & 0 & 0 & c_{1} \\
a_{2} & 1 & 0 & 0
\end{array}\right)\left(\begin{array}{cccc}
c_{3} c_{4}+b_{4} & 0 & 1 & c_{3} \\
c_{4} & 0 & 0 & 1 \\
c_{3} c_{4}+1 & 0 & 0 & c_{3} \\
a_{4} & 1 & 0 & 0
\end{array}\right)
$$

has the eigenvalue $\varrho_{1}=1$.

(ii) We consider the family of examples given by

$$
x=\left(\begin{array}{llllll}
1 & 0 & 1 & 0 & 2 & 3 \\
0 & 1 & 0 & 1 & 0 & 4 \\
1 & 3 & 1 & 2 & 2 & c_{6}
\end{array}\right) .
$$

Then

$$
B=\left(\begin{array}{cccc}
15 & 1 & 4 & 5 \\
9 & 1 & 3 & 3 \\
12 & 1 & 4 & 4 \\
2 & 0 & 0 & 1
\end{array}\right)\left(\begin{array}{cccc}
2 c_{6}+4 & 0 & 1 & 2 \\
c_{6} & 0 & 0 & 1 \\
2 c_{6}+1 & 0 & 0 & 2 \\
3 & 1 & 0 & 0
\end{array}\right)
$$

A calculation shows

$$
f(-1)=\operatorname{det}(\mathbf{1}+B)=4\left(c_{6}-15\right) .
$$

If $c_{6}=15$ then $f(-1)=0$. If we take $5 \leq c_{6} \leq 14$, then $f(-1)<0$. If we choose $c_{6} \geq 16$ then $f(-1)>0$.

Remarks. (1) Dubois, Farhane \& Paysant-Le Roux [1] give an example with $\varrho_{1}>1$ for period length $p=3$, namely the periodic algorithm

$$
x=\left(\begin{array}{ccc}
1 & b & b \\
b & 1 & b \\
b+1 & b & b
\end{array}\right)
$$

with $b \geq 6$.

(2) It is easy to exhibit examples with $\left|\varrho_{1}\right|>1$ in dimension $d=3$ for the Brun algorithm. The 3-dimensional Brun algorithm is governed by the matrices

$$
\beta(t)=\left(\begin{array}{llll}
t & 1 & 0 & 0 \\
1 & 0 & 0 & 0 \\
0 & 0 & 1 & 0 \\
0 & 0 & 0 & 1
\end{array}\right), \quad \gamma(t)=\left(\begin{array}{cccc}
t & 0 & 1 & 0 \\
1 & 0 & 0 & 0 \\
0 & 1 & 0 & 0 \\
0 & 0 & 0 & 1
\end{array}\right), \quad \delta(t)=\left(\begin{array}{cccc}
t & 0 & 0 & 1 \\
1 & 0 & 0 & 0 \\
0 & 1 & 0 & 0 \\
0 & 0 & 1 & 0
\end{array}\right) .
$$


They correspond to the branches

$$
\begin{aligned}
& T\left(x_{1}, x_{2}, x_{3}\right)=\left(\frac{1}{x_{1}}-t, \frac{x_{2}}{x_{1}}, \frac{x_{3}}{x_{1}}\right), \\
& T\left(x_{1}, x_{2}, x_{3}\right)=\left(\frac{x_{2}}{x_{1}}, \frac{1}{x_{1}}-t, \frac{x_{3}}{x_{1}}\right), \\
& T\left(x_{1}, x_{2}, x_{3}\right)=\left(\frac{x_{2}}{x_{1}}, \frac{x_{3}}{x_{1}}, \frac{1}{x_{1}}-t\right),
\end{aligned}
$$

where $t=\left[1 / x_{1}\right]$. Then

$$
\beta(1)^{3} \delta(1) \gamma(1)=\left(\begin{array}{cccc}
5 & 0 & 5 & 3 \\
3 & 0 & 3 & 2 \\
1 & 0 & 0 & 0 \\
0 & 1 & 0 & 0
\end{array}\right)
$$

and

$$
f(\varrho)=\varrho^{4}-5 \varrho^{3}-7 \varrho^{2}+\varrho+1,
$$

which implies $f(-1)=-1$ and therefore $\varrho_{1}<-1$ (Toussaint [9]).

4. The Paley-Ursell constant. Paley \& Ursell [3] state without proof that for any dimension $d \geq 3$ there is a constant $\omega=\omega(d)$ such that $\left|D_{N}\right| \leq \omega^{N}$. In the following theorem we give an estimate for this constant in dimension $d=3$.

TheOREM 4. Let $\omega$ be the greatest root of $\omega^{3}=\omega^{2}+1$. Then $\left|D_{N}\right| \leq \omega^{N}$.

REMARK. If the algorithm is periodic with period length $p$ and if $\varrho_{1}$ is the second eigenvalue then $\left|\varrho_{1}\right| \leq \omega^{p}$.

Proof. The starting point is the relation

$$
D_{N+4}=-y_{1} D_{N+1}-y_{2} D_{N+2}-y_{3} D_{N+3} .
$$

Note that $0 \leq y_{i} \leq 1, i=1,2,3$. Let us assume that $D_{N+4}>0$. Then we distinguish the following cases.

(1) If $D_{N+3}<0, D_{N+2}<0, D_{N+1}<0$ then we use additionally the recursion

$$
D_{N+4}=a_{3}^{(N)} D_{N+3}+a_{2}^{(N)} D_{N+2}+a_{1}^{(N)} D_{N+1}+D_{N},
$$

which immediately shows that $D_{N}>0$ and therefore $\left|D_{N+4}\right| \leq\left|D_{N}\right|$.

(2) This case is further subdivided:

(2.1) $D_{N+3}<0, D_{N+2}>0, D_{N+1}>0$. Here we obtain

$$
D_{N+4} \leq y_{3}\left|D_{N+3}\right| \leq\left|D_{N+3}\right| \text {. }
$$


(2.2) $D_{N+3}>0, D_{N+2}<0, D_{N+1}>0$. In this case we get

$$
D_{N+4} \leq y_{2}\left|D_{N+2}\right| \leq\left|D_{N+2}\right| \text {. }
$$

(2.3) $D_{N+3}>0, D_{N+2}>0, D_{N+1}<0$. In a similar way we get

$$
D_{N+4} \leq y_{1}\left|D_{N+1}\right| \leq\left|D_{N+1}\right| \text {. }
$$

Therefore the quantity $\left|D_{N}\right|$ can only grow in one of the remaining cases.

(3) This case is again subdivided:

(3.1) $D_{N+3}>0, D_{N+2}<0, D_{N+1}<0$. Then we get

$$
D_{N+4} \leq\left|D_{N+2}\right|+\left|D_{N+1}\right| \text {. }
$$

(3.2) $D_{N+3}<0, D_{N+2}>0, D_{N+1}<0$. This leads to

$$
D_{N+4} \leq\left|D_{N+3}\right|+\left|D_{N+1}\right| \text {. }
$$

(3.3) $D_{N+3}<0, D_{N+2}<0, D_{N+1}>0$. In this case we obtain

$$
D_{N+4} \leq\left|D_{N+3}\right|+\left|D_{N+2}\right| \text {. }
$$

Note that the results (1) to (3) are also valid if all signs are reversed. However, the estimates in (3.2) and (3.3) can be ameliorated when we consider the sign of $D_{N}$.

(3.2.1) Let $D_{N}>0$. Then from (3) we get $\left|D_{N+3}\right| \leq\left|D_{N+2}\right|+D_{N}>0$ and therefore

$$
D_{N+4} \leq\left|D_{N+2}\right|+\left|D_{N+1}\right|+\left|D_{N}\right| .
$$

(3.2.2) Let $D_{N}<0$. Then from (2) we obtain $\left|D_{N+3}\right| \leq\left|D_{N+2}\right|$ and eventually

$$
D_{N+4} \leq\left|D_{N+2}\right|+\left|D_{N+1}\right| .
$$

(3.3.1) Let $D_{N}>0$. Then again by (3) we obtain $\left|D_{N+3}\right| \leq\left|D_{N+1}\right|+D_{N}$ and

$$
D_{N+4} \leq\left|D_{N+2}\right|+\left|D_{N+1}\right|+\left|D_{N}\right| .
$$

(3.3.2) Let $D_{N}<0$. Then by (2), $\left|D_{N+3}\right| \leq\left|D_{N+1}\right|$ and

$$
D_{N+4} \leq\left|D_{N+2}\right|+\left|D_{N+1}\right| \text {. }
$$

If we now assume by induction that $D_{N} \leq \omega^{N}$ then the worst cases (3.2.1) and (3.3.1) lead to

$$
\omega^{N+4} \leq \omega^{N+2}+\omega^{N+1}+\omega^{N} .
$$

Therefore the greatest root of $\omega^{4}=\omega^{2}+\omega+1$ gives the required estimate, but note that $\omega^{4}-\omega^{2}-\omega-1=(\omega+1)\left(\omega^{3}-\omega^{2}-1\right)$.

REMARK. We note that $1<\omega \sim 1.465<(1+\sqrt{5}) / 2$. 


\section{References}

[1] E. Dubois, A. Farhane and R. Paysant-Le Roux, The Jacobi-Perron Algorithm and Pisot numbers, Acta Arith. 111 (2004), 269-275.

[2] C. G. J. Jacobi, Allgemeine Theorie der kettenbruchähnlichen Algorithmen, in welchen jede Zahl aus drei vorhergehenden gebildet wird, J. Reine Angew. Math. 69 (1868), 29-64.

[3] R. E. A. C. Paley and H. D. Ursell, Continued fractions in several dimensions, Proc. Cambridge Philos. Soc. 26 (1930), 127-144.

[4] R. Paysant-Le Roux et E. Dubois, Une application des nombres de Pisot à l'algorithme de Jacobi-Perron, Monatsh. Math. 98 (1984), 145-155.

[5] O. Perron, Grundlagen für eine Theorie des Jacobischen Kettenbruchalgorithmus, Math. Ann. 64 (1907), 1-76.

[6] W. Schmidt, Flächenapproximation beim Jacobialgorithmus, ibid. 136 (1958), 365374.

[7] F. Schweiger, Der Satz von Hurwitz beim Jacobialgorithmus, Monatsh. Math. 81 (1975), 215-218.

[8] - Multidimensional Continued Fractions, Oxford Univ. Press, Oxford, 2000.

[9] H.-J. Toussaint, Der Algorithmus von Viggo Brun und verwandte Kettenbruchentwicklungen, Dissertation, Tech. Univ. München, 1986.

Department of Mathematics

University of Salzburg

Hellbrunnerstrasse 34

A-5020 Salzburg, Austria

E-mail: fritz.schweiger@sbg.ac.at

Received on 20.8.2007

and in revised form on 9.5.2008 